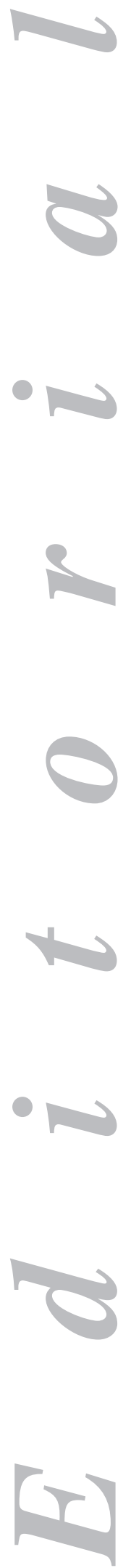

\title{
Morbidity associated with the management of rectal cancer and its impact on patient quality of life
}

Around $75 \%$ of patients with rectal cancer may be surgically treated with an intention to cure. The goal of curative surgery in rectal cancer is tumor resection with adequate margins, which is addressed by performing a wide excision of the underlying soft tissue and associated lymph nodes. This implies an extension of tumor resection margins to at least $5 \mathrm{~cm}$ around the involved area. The surgical approach to rectal cancer considers the neoplasm's location -upper ( 12 to $16 \mathrm{~cm}$ ), middle ( 7.5 to 12 $\mathrm{cm}$ ) or lower and anal canal (less than $7.5 \mathrm{~cm}$ ); the distance from the tumor's lower border to the linea dentata is the most significant factor when it comes to the selection of a procedure type. The standard management of this type of cancer is radical resection, either lower anterior resection or abdominoperineal amputation, depending on the tumor's upper-mid location or lower-mid location, respectively (1).

In the past few years significant improvements in surgical technique and technology itself have been made mostly regarding the development of suturing devices, which allowed a greater number of procedures with sphincter preservation, ultralow colorectal anastomosis, and coloanal anastomosis. A number of low tumors still require abdominoperineal amputation, however. Despite the aforementioned advances, rectal cancer surgery is burdened with the fact that these techniques are associated with high morbidity even in experienced hands, which results from anastomotic dehiscence, genitourinary dysfunction, fecal incontinence, permanent colostomy, etc. In addition, local recurrence develops in $15-50 \%$ of patients after conventional surgery, even following complete resection (2). Local recurrence is associated with positive resection margins, histologic differentiation grade, rectal wall invasion $(\mathrm{T})$, and nodal infiltration $(\mathrm{N})$.

Therefore we envisage two interrelated groups of difficulties deriving from curative-intent surgery for rectal cancer: the morbidity arising from surgical techniques, which directly affects quality of life, and the potential for local recurrence of disease, which clearly relates to patient survival and, through therapies intended to prevent such recurrence, to morbidity and quality of life involving these subjects.

Health is currently understood as a complete state of physical, mental, and social well-being, rather than merely an absence of disease (3). The fact of suffering from disease results in a multidimensional involvement of life, with changes in the personal, familiar, and social spheres. Thus, not only objective data such as mortality rate should be considered when assesing a disorder's impact, but we should rather take into account the impact of disease on the subject's self-perceived health, that is, on the subject's quality of life. Quality of life is considered a subjective and dynamic characteristic of individuals, and is assessed by using various techniques such as personal interviewing and questionnaires. Measuring quality of life allows an as- 
sessment of procedure outcomes, of the personal, familiar, and social impact of disease, and of quality of life; it also allows to improve our understanding of disease, to identify health-care needs, etc. (4).

In this issue of the Revista Española de Enfermedades Digestivas, Pérez FJ et al. (5) discuss the results of a study on the factors influencing quality of life in patients with locally advanced rectal cancer undergoing surgery. For their assessment they used the Nottingham Health Profile, a generic questionnaire, and QLQCR 38, a validated questionnaire specific for patients with colorectal cancer that was modified from the QLQ-CR 30 questionnaire, which is administered to patients with cancer in general. This questionnaire includes two modules -functional and symptomatic.

From the study of the factors included in these questionnaires Pérez FJ et al. concluded that women, patients with mid-third rectal tumors, and patients undergoing lower anterior resection perceived their quality of life as more negatively changed following rectal cancer surgery. The latter two factors are clearly interrelated, since this type of resection is commonly applied for tumors in this location. The authors report that surgical technique is the only factor that may be modified in order to improve quality of life for this group of patients. Abdominoperineal amputation includes a resection of the whole rectum and anal canal, the mesorectum, most anusraising muscles, ischiorectal fat, upper hemorrhoidal and lower mesenteric vessels, and the corresponding left colon and mesocolon portion. With this surgical technique, rectal dissection may damage the pelvic autonomic plexus, and thus impair sexual and urinary functions. During lower anterior resection sphincters may be preserved and permanent colostomy avoided, thus decreasing the potential development of impotence, impaired ejaculation, urine retention, etc. Notwithstanding, trauma to the anal sphincter as induced upon stapler introduction may result in incontinence, defecatory urgency, etc.

The authors report that expectations also differ amongst patients, which may have influenced results obtained: in abdominoperineal amputation, patients assume their need for colostomy and become psychologically adapted to it once they check their activity remains unrestricted; patients undergoing lower anterior resection expect that their usual intestinal and sphincter function will be preserved, and feel deeply let down when difficulties regarding fecal incontinence arise. To avoid this, thoroughly informing patients on either technique seems relevant enough prior to surgery.

Anyway, considering the benefits and shortcomings of both techniques, the goal of surgery is to ensure oncologic safety besides modifying factors apt to negatively influence quality of life following the procedure. As previously mentioned, we have been witness to a great technical advance in the surgery of rectal cancer, but issues regarding surgical technique remain that require refinement in order to reduce morbidity and hence improve quality of life for operated patients. A surgical option for rectal cancer that is associated with decreased morbidity is transanal endoscopic surgery, which allows the preservation of sexual, vesical, and anal functions. This curative-intent local resection technique is indicated for patients with well- or moderately-differentiated tumors confined to the rectal wall, not extending beyond the muscularis layer, and lacking nodal involvement (T0-T1, N0) $(6,7)$. In carefully selected patients, recurrence and survival rates with this therapeutic modality are similar to those of radical surgery (8). Success with this surgical technique definitely depends on patient triage, and a thorough preoperative study including endorectal endoscopy is therefore mandatory. 
Alternatively, regarding local rectal cancer recurrence, Garay M et al. (9) studied the morbidity of postoperative radiotherapy in operated individuals. These authors encountered that postoperative radiotherapy decreased the potential for local recurrence in rectal cancer, but at the expense of increased acute and late morbidity. As goals for rectal cancer management, they claim that not only an increase in total and free-of-disease survival, and a reduction in local recurrence and postoperative complications should be considered, but also that these criteria are met while preserving the best quality of life for patients. In view of their results, they suggested a reassessment of postoperative radiotherapy, which has a negative impact on patient quality of life, and a consideration of other similarly highly effective options to reduce local recurrence rates in rectal cancer with less morbidity: total mesorectal excision and preoperative chemoradiotherapy.

The mesorectum consists of fatty, lymphovascular, and neural tissues around the rectum, and its significance in rectal cancer results from its potential neoplastic involvement, either from direct extension or lymph node infiltration, or in the form of isolated implants away from the primary tumor. Distant metastases develop in the mesorectum in 4 to $20 \%$ of patients. A close relationship exists between the extent of mesorectal involvement and prognosis following surgery. Thus, total mesorectal excision is postulated in curative-intent surgery for rectal cancer, especially for mid- to lower-third neoplasms. In expert hands, total mesorectal excision allows a reduction of local recurrence rates to levels below 10\%, as well as an improvement in survival for patients with this neoplasm (10). Some authors consider that total mesorectal excision may adequately decrease local recurrence rates, so that preoperative radiotherapy would be rendered unnecessary for a high number of patients (11). However, other studies have drawn differing conclusions.

The preoperative use of chemoradiotherapy followed by total mesorectal excision is currently accepted as the treatment of choice for patients with mid- and lower-third rectal cancer in whom mesorectal and/or nodal infiltration is suspected in the extension study. The benefits of preoperative radiotherapy include easier tumoral resection, decreased risk for tumor cell spread from surgical management, increased potential for anal sphincter preservation, and lower morbidity versus postoperative radiotherapy (small intestinal loop radiation is avoided to prevent fixation following surgery), with higher tumoral radiosensitivity (vascular changes occur during surgery, which may entail poorer tumoral oxygenation). Preoperative radiotherapy provides additional benefits when administered to patients undergoing adequate mesorectal resection, and significantly reduces local recurrence rates when compared to conventional surgery alone (12).

To conclude, radical surgery techniques for rectal cancer currently result in reduced quality of life for patients. Local resection using transanal endoscopic surgery may be considered a therapeutic modality with less morbidity, and its success directly depends upon a careful triage of patients with early-stage tumors. The prevention of local recurrence in rectal cancer using preoperative chemoradiotherapy plus mesorectal excision during surgery is more effective than postoperative radiotherapy and impairs patient quality of life to a lesser extent. 


\section{References}

1. Eckhauser FE, Knol JA. Surgery for primary and metastatic colorectal cancer. Gastroenterol Clin North Am 1997; 26: 103-27.

2. Kapiteijn E, Marijnen C, Colenbrander A, et al. Local recurrence in patients with rectal cancer diagnosed between 1988 and 1992: a population-based study in the west Netherlands. Eur J Surg Oncol 1998; 24: 528-35.

3. Casellas F, López J. Evaluación de la calidad de vida en las enfermedades digestivas. Gastroenterol Hepatol 2004; 27: 58-68.

4. Badía X. Qué es y cómo se mide la calidad de vida relacionada con la salud. Gastroenterol Hepatol 2004; (Supl. 27): 2-6.

5. Pérez Lara FJ, Navarro Piñero A, De la Fuente Perucho A. Estudio de factores de calidad de vida en los pacientes con cáncer de recto localmente avanzado. Rev Esp Enferm Dig 2004; 96 (11): 746-57.

6. Mellgren A, Sirivongs P, Rothenberger DA, Madoff R, García Aguilar J. Is local excision adequate therapy for early rectal cancer? Dis Colon Rectum 2000; 43: 1064-74.

7. Visser C, Varma MG, Welton ML. Local therapy for rectal cancer. Surg Oncol 2001; 10: 61-9.

8. Winde G, Nottberg H, Keller R, Schmid K, Bünte H. Surgical cure for early rectal carcinomas (T1): transanal endoscopic microsurgery vs anterior resection. Dis Colon Rectum 1996; 39: 969-76.

9. Garay Burdeos M, García-Botella M, Viciano Pascual V, Torregrosa Macías MD, Aguiló Lucía J, Ata M, et al. Morbilidad de la radioterapia postoperatoria en el cáncer de recto. Rev Esp Enferm Dig 2004; 96 (11): 765-72

10. Pera M. Cáncer de recto: valor de la resección del mesorrecto en el pronóstico de la enfermedad. Gastroenterol Hepatol 2003; 26: 159-60.

11. Simunovic M, Sexton R, Rempel E, Moran BJ, Heald RJ. Optimal preoperative assessment and surgery for rectal cancer may greatly limit the need for radiotherapy. Br J Surg 2003; 90: 999-1003.

12. Kapiteijn E, Marijnen CA, Nagtegaal ID, Putter H, Steup WH, Wiggers T, et al. Preoperative radiotherapy combined with total mesorectal excision for resectable rectal cancer. N Engl J Med 2001; 345: 638-46.

\section{Morbilidad del tratamiento del cáncer de recto y repercusión sobre la calidad de vida de los pacientes}

Alrededor del $75 \%$ de los pacientes con cáncer de recto puede ser tratado con cirugía con intención curativa. El objetivo de la cirugía curativa del cáncer de recto es la extirpación del tumor con un margen adecuado, realizando una amplia escisión del área de tejido blando adyacente y los ganglios linfáticos asociados. Esto implica la ampliación del margen de resección del tumor al menos $5 \mathrm{~cm}$ sobre la zona afectada. El abordaje quirúrgico del cáncer de recto se realiza atendiendo a la localización superior (12 a $16 \mathrm{~cm}$ ), media $(7,5 \mathrm{a} 12 \mathrm{~cm}$ ) o inferior y de canal anal (a menos de $7,5 \mathrm{~cm}$ ) de la neoplasia, siendo la distancia entre el límite inferior del tumor y la línea dentada el factor más importante en la elección del tipo de intervención. El tratamiento estándar para este tipo de cáncer es la resección radical, ya sea la resección anterior baja o la amputación abdominoperineal, según se trate de tumores de localización superior-media o bien media-inferior, respectivamente (1).

En los últimos años se han introducido importantes mejoras en la técnica quirúrgica y en la propia tecnología, principalmente en el desarrollo de los dispositivos de sutura, lo cual ha permitido realizar un mayor número de intervenciones con preservación de esfínteres, anastomosis colorrectales ultrabajas o anastomosis coloanales. No obstante, algunos tumores bajos continúan requiriendo la realización de amputaciones abdominoperineales. A pesar de los avances mencionados, la cirugía del cáncer de recto está gravada por el hecho de que estas técnicas se asocian a una elevada morbilidad, incluso en manos expertas, motivada por la aparición de dehiscencia anastomótica, disfunción genitourinaria, incontinencia fecal, colostomía permanen- 
te, etc. Además, aunque se realice una resección completa, la enfermedad recidiva localmente en un $15-50 \%$ de los pacientes después de la cirugía convencional (2). La recidiva local está relacionada con la positividad de los márgenes de resección, el grado de diferenciación histológica, la invasión de la pared rectal (T) y la infiltración ganglionar $(\mathrm{N})$.

Nos encontramos, por lo tanto, con dos grupos de problemas derivados de la cirugía con intención curativa del cáncer de recto relacionados entre sí: la morbilidad de las técnicas quirúrgicas, que afecta directamente a la calidad de vida de los pacientes, y la posibilidad de recidiva local de la enfermedad, en clara relación con la supervivencia de los pacientes intervenidos y, a través de los tratamientos aplicados para evitar dicha recidiva, con la morbilidad y la calidad de vida de estos sujetos.

La salud se entiende, hoy día, como el estado completo de bienestar físico, mental y social, y no sólo como la ausencia de enfermedad (3). El hecho de padecer una enfermedad provoca una afectación mutidimensional de la vida, con cambios en las esferas personal, familiar y social. Por ello, a la hora de evaluar el impacto derivado de una determinada enfermedad no sólo hay que tener en cuenta datos objetivos, como la mortalidad, sino que deberemos considerar la repercusión que tiene la enfermedad sobre la percepción de salud del sujeto, es decir, sobre su calidad de vida. La calidad de vida se considera como una característica subjetiva y dinámica del individuo, y su evaluación se lleva a cabo mediante técnicas diversas, como son la entrevista personal y los cuestionarios. La medición de la calidad de vida permite valorar el resultado de una intervención, el impacto personal, familiar y social de la enfermedad, y la calidad de la asistencia; mejorar los conocimientos de una enfermedad; identificar necesidades sanitarias, etc. (4).

En este número de la Revista Española de Enfermedades Digestivas, Pérez FJ y cols. (5), exponen los resultados de un estudio sobre los factores que influyen en la calidad de vida de los pacientes con cáncer de recto localmente avanzado que son sometidos a cirugía. Para realizar su evaluación utilizan el perfil de salud de Nottingham, que es un cuestionario genérico, y el QLQ-CR 38, un cuestionario validado específico para pacientes con cáncer colorrectal, modificado del modelo QLQCR 30 que se empleó en pacientes con cáncer en general. Este cuestionario consta de dos módulos: funcional y de síntomas.

Del estudio de los factores incluidos en estos cuestionarios, Pérez FJ y cols. concluyen que tras la cirugía del cáncer de recto ven más modificada en sentido negativo su calidad de vida las mujeres, los pacientes con tumores localizados en el tercio medio rectal y aquellos sometidos a resección anterior baja. Los dos últimos factores están claramente relacionados, puesto que este tipo de resección suele aplicarse en los tumores de dicha localización. Los autores refieren que el único factor modificable para mejorar la calidad de vida en este grupo de pacientes es la técnica quirúrgica. La amputación abdominoperineal incluye la resección completa del recto y del canal anal, el mesorrecto, buena parte de la musculatura elevadora del ano, la grasa isquiorrectal, los vasos hemorroidales superiores y mesentéricos inferiores y la porción de colon izquierdo correspondiente con su meso. Con esta técnica quirúrgica, la disección del recto puede dañar el plexo autonómico pélvico, dando lugar a una alteración de las funciones sexual y urinaria. En el caso de la resección anterior baja pueden preservarse los esfínteres, evitando la colostomía permanente, con menor posibilidad de aparición de impotencia, dificultades eyaculatorias, retención de orina, etc. No obstante, el traumatismo provocado sobre el esfínter anal para introducir la grapadora puede dar lugar a incontinencia, urgencia defecatoria, etc. 
Exponen los autores que las expectativas de los pacientes son igualmente diferentes y ello ha podido influir en los resultados que obtienen: en los casos de amputación abdominoperineal, los pacientes asumen la necesidad de colostomía y se adaptan psicológicamente a ella una vez que observan que no limita su actividad; los pacientes sometidos a una resección anterior baja esperan mantener la función intestinal y esfinteriana habitual, sufriendo una importante decepción cuando aparecen los problemas de incontinencia fecal. Para evitar esto, parece relevante que la información a los pacientes previa a la cirugía sea lo más extensa posible, ya sea respecto a una técnica quirúrgica u otra.

El fin de la cirugía, en cualquier caso, y valorando las ventajas e inconvenientes de ambas técnicas, es garantizar la seguridad oncológica atendiendo, además, a modificar aquellos factores que pueden influir negativamente en la calidad de vida del paciente tras la cirugía. Como se mencionó previamente, hemos asistido a un gran avance técnico en la cirugía del cáncer de recto, pero todavía hay cuestiones de la técnica quirúrgica que deben perfeccionarse con el fin de reducir la morbilidad y, con ello, mejorar la calidad de vida de los pacientes intervenidos. Una opción quirúrgica para el cáncer de recto con menor morbilidad es la cirugía transanal endoscópica, con la cual es posible preservar las funciones sexual, vesical y anal. Esta técnica de resección local con intención curativa está indicada en pacientes con tumores confinados a la pared rectal que no sobrepasen la muscular, bien o moderadamente diferenciados y sin afectación ganglionar (T0-T1, N0) (6,7). En pacientes seleccionados cuidadosamente, la tasa de recidivas y supervivencia con esta modalidad terapéutica son similares a las de la cirugía radical (8). Hay que destacar que para conseguir el éxito con esta técnica quirúrgica es crítica la selección de los pacientes y, por ello, es fundamental realizar un exhaustivo estudio preoperatorio que incluya ecoendoscopia endorrectal.

Por otra parte, en relación con la recidiva local del cáncer de recto, Garay M y cols. (9), analizan la morbilidad de la radioterapia postoperatoria en los individuos intervenidos. Estos autores encuentran que la radioterapia postoperatoria reduce la posibilidad de recidiva local del cáncer de recto, pero ello es a consta de incrementar la morbilidad de los pacientes, tanto de forma aguda como de forma tardía. Como objetivos en el tratamiento del cáncer de recto, plantean no sólo incrementar la supervivencia total y libre de infermedad, y reducir la tasa de recidiva local y las complicaciones postoperatorias, sino intentar conseguir estos requisitos con la mejor calidad de vida para el paciente. Teniendo en cuenta sus resultados, proponen reevaluar la indicación de la radioterapia postoperatoria, que influye negativamente en la calidad de vida del paciente, y considerar otras opciones que han mostrado una elevada y similar eficacia en la reducción de la tasa de recidiva local del cáncer de recto, con una menor morbilidad: la excisión total del mesorrecto y la quimiorradioterapia preoperatoria.

El mesorrecto es el tejido graso, linfovascular y nervioso que rodea al recto, y su importancia en el cáncer de recto se debe a que puede estar afectado por la neoplasia, ya sea mediante extensión directa, infiltración de los ganglios linfáticos o en forma de depósitos aislados alejados del tumor primario. Aparecen metástasis en el mesorrecto alejadas del tumor primario en un porcentaje que varía entre el 4 y el $20 \%$ de los casos. Existe una estrecha relación entre el grado de afectación del mesorrecto y el pronóstico tras la intervención quirúrgica. Por ello, en la cirugía del cáncer de recto con intención curativa se propugna la inclusión de la exéresis total del mesorrecto, especialmente en los tumores del tercio medio e inferior. En manos expertas, la excisión total de mesorrecto ha permitido reducir los porcentajes de re- 
currencia local por debajo del $10 \%$ y mejorar la supervivencia de los pacientes con esta neoplasia (10). Algunos autores consideran que la excisión total del mesorrecto puede reducir los porcentajes de recurrencia local de forma suficiente, de tal manera que la radioterapia preoperatoria no sería necesaria en un gran número de pacientes (11). Sin embargo, otros estudios han obtenido conclusiones diferentes.

En la actualidad se admite la aplicación de quimiorradioterapia preoperatoria seguida de excisión total del mesorrecto como tratamiento de elección en pacientes con cáncer de recto del tercio medio e inferior con sospecha, según el estudio de extensión, de infiltración del mesorrecto y/o ganglios linfáticos. Entre las ventajas de la radioterapia preoperatoria se encuentran el que facilita la resección tumoral, disminuye el riesgo de diseminación de células tumorales por la manipulación quirúrgica, aumenta la posibilidad de preservar el esfínter anal, y que presenta menor morbilidad que la radioterapia postoperatoria (se evita irradiar asas de intestino delgado que pueden quedar fijas tras la cirugía), con una mayor radiosensibilidad tumoral (con la cirugía se producen modificaciones vasculares que pueden conllevar una peor oxigenación tumoral). La radioterapia preoperatoria proporciona un beneficio adicional cuando se administra a aquellos pacientes a quienes se les practica una adecuada exéresis del mesorrecto, disminuyendo el porcentaje de recurrencia local de forma significativa en relación con la cirugía convencional aplicada de forma aislada (12).

Como conclusión, las técnicas de cirugía radical del cáncer de recto suponen, hoy en día, un menoscabo en la calidad de vida de los pacientes. La resección local mediante cirugía transanal endoscópica puede considerarse la modalidad terapéutica con menor morbilidad, cuyo éxito depende directamente de la selección cuidadosa de los pacientes con tumores en estadios tempranos. La prevención de la recidiva local del cáncer de recto mediante la aplicación de quimiorradioterapia preoperatoria y la inclusión de la excisión del mesorrecto en el acto quirúrgico resulta más eficaz y altera en menor grado la calidad de vida de los pacientes que la radioterapia postoperatoria.

M. L. Manzano Alonso

Servicio de Medicina de Aparato Digestivo. Hospital 12 de Octubre. Madrid 